\title{
Performance of a High-Resolution Digital Positron-Lifetime Spectrometer
}

\author{
F. BEČVÁ̌̌, J. ČížEK ANd I. PROCHÁzKA \\ Faculty of Mathematics and Physics, Charles University in Prague \\ V Holešovičkách 2, Praha 8, CZ-180 00, Czech Republic \\ This work describes a digital positron-lifetime spectrometer based on \\ two ultrafast digitizers. Performance of the digital spectrometer in conditions \\ of a routine measurement is compared with an analogue setup connected to \\ the same detectors. It was found that engagement of the digital setup leads \\ to an improvement of timing resolution (FWHM for ${ }^{22} \mathrm{Na}$ ) from $169 \mathrm{ps}$ for the \\ traditional analogue setup to $146 \mathrm{ps}$ for the digital setup. The coincidence \\ count rate for $1.2 \mathrm{MBq}^{22} \mathrm{Na}$ source is $75-80$ coinc./s for both configurations. \\ These results clearly demonstrate the advantages of the digital timing over \\ the traditional analogue setup.
}

PACS numbers: 78.70.Bj

\section{Introduction}

Timing resolution power is one of the most important parameters of any positron-lifetime (PL) spectrometer. Recent development in the sampling rate of ultrafast digitizers opened a new possibility for timing, called digital timing. The detector pulses are digitized in real time by an ultrafast digitizer which replaces all the analogue timing electronics. The digitized signals (wave forms) are stored in computer and timing information is derived off-line by software. This new digital approach has several undisputable advantages: (i) all detector signals are directly accessible for the analysis, (ii) the timing analysis can be repeated many times in order to find the optimal strategy how to derive the timing information, and (iii) time consuming adjustment of the analogue nuclear instrument module (NIM) devices is not necessary anymore.

However, it is not clear yet whether the digital setup provides better timing resolution than the traditional analogue setup. Digital PL spectrometer equipped with ultrafast digitizers connected to detectors with plastic scintillators was described in Ref. [1]. The authors achieved moderate timing resolution of $\approx 200 \mathrm{ps}$ and reported no improvement in timing resolution compared to the analogue setup. Saito et al. [2] achieved excellent timing resolution of 144 ps by employing fast digital oscilloscope connected to detectors equipped with $\mathrm{BaF}_{2}$ scintillators. However, no comparison with the analogue setup has been done. It has to be mentioned that 
similar timing resolution of 143 ps was obtained with traditional analogue setup [3]. To clarify whether digital timing improves timing resolution power or not, it is necessary to compare performance of digital and analogue setup connected to identical detectors. Such comparison was performed in this work.

\section{Experimental}

Detectors consist of Hamamatsu H3378 photomultipliers optically coupled with truncated cone $(\varnothing 18-36 \mathrm{~mm}$, height $12 \mathrm{~mm}) \mathrm{BaF}_{2}$ scintillators. The detector pulses were taken from anode using a standard high voltage divider supplied by the producer. A $1.2 \mathrm{MBq}{ }^{22} \mathrm{Na}_{2} \mathrm{CO}_{3}$ positron source was deposited on a $2 \mu \mathrm{m}$ thick Mylar foil. A well-annealed $\alpha$-Fe $(99.999 \%)$ was used as a reference specimen.

A schematic diagram of the analogue and digital setup is shown in Fig. 1A and $\mathrm{B}$, respectively. In digital setup, the detector pulses were divided into two parts

(A)
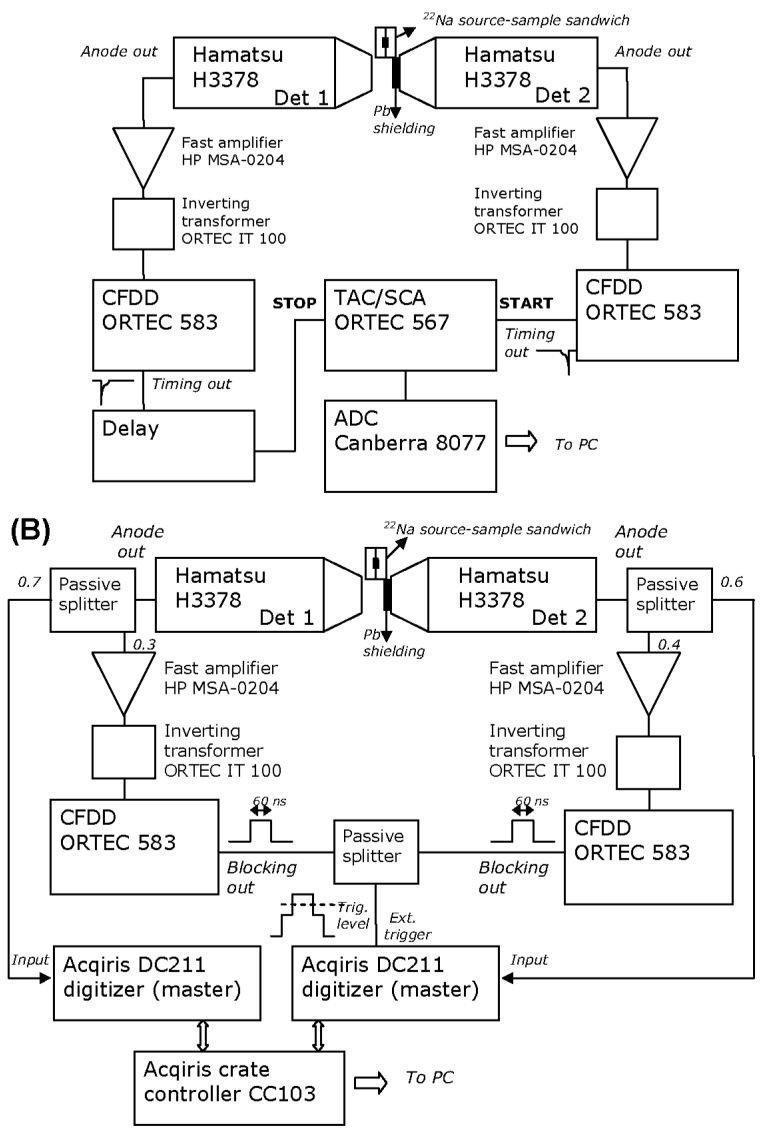

Fig. 1. A schematic diagram of (A) the analogue setup, (B) the digital setup. 
by impedance balanced passive splitters. The larger parts of signals were led to input channels of two ultrafast 8-bit digitizers Acqiris DC 211 with the sampling rate $4 \mathrm{GS} / \mathrm{s}$ working at a common crystal controlled time base. The smaller fractions of signals were amplified by HP MSA-0204 amplifiers to feed constant fraction differential discriminators (CFDD)'s Ortec 583. The rectangular blocking out signals with duration of $\approx 60$ ns produced by CFDD's were passively mixed to form an input signal for external triggering the digitizers. The discrimination level was adjusted so that only coinciding detector signals are processed. Further restriction is imposed by adjusting the lower level of CFDD's pulse height window to a level corresponding to $\gamma$-ray energy of $\approx 380 \mathrm{keV}$. Contrary to the analogue setup, CFDD's are not used for timing. Their purpose is to ensure that only coincidence events are digitized while uninteresting single track events are filtered out.

Each acquired wave form was formed by 300 sample points with spacing of $250 \mathrm{ps}$ covering thus a time interval of $75 \mathrm{~ns}$. The wave forms were subsequently analyzed off-line by integral constant fraction (iCF) method developed by us [4]. The optimal iCF level for our wave forms was found to be $7 \%$. The shape of the integrated wave forms was controlled by digital filters [4] which excluded wave forms with distorted shape. The energy windows imposed on the digitized wave forms were $1080-1550 \mathrm{keV}$ and $460-590 \mathrm{keV}$ for the start and the stop signal, respectively. The software analysis was performed in two modes (i) START-STOP (det 1 is "start detector" and det 2 "stop detector"), and (ii) STOP-START mode, where the roles of detectors were reversed. Application of these two modes doubles the count rate at no cost. The results obtained in START-STOP and STOP-START mode are practically identical. Thus, we always used averaged results obtained from fitting of the START-STOP and the STOP-START mode PL spectra.

\section{Results and discussion}

The results of PL measurements with the reference $\alpha$-Fe specimen are collected in Table. The $\alpha$-Fe specimen exhibits a free positron component with lifetime $\tau_{1}=107 \mathrm{ps}$ which agrees well with the bulk Fe lifetime [5]. Two additional weak components with lifetimes $\tau_{2}$ and $\tau_{3}$ come from positron annihilations inside the positron source and in the covering foils. The resolution function is well described by two Gaussians. The timing resolution achieved in the START-STOP (see Fig. 2) and STOP-START mode, respectively, is 145 and 147 ps.

Virtually, the same lifetimes and intensities as those in digital setup were obtained also in the analogue setup measurement. The resolution functions for the digital and analogue setup are compared in the inset in Fig. 2. The widths of the two main Gaussians and their separation in the analogue setup are larger than in the digital setup. In addition, a weak and broad third Gaussian, which accounts for pile-up effects, must be added to describe well the resolution function. Timing resolution in the analogue setup is 169 ps. Thus, timing resolution achieved in the digital setup is about of 23 ps better than in the corresponding analogue 
TABLE

Comparison of digital and analogue setup performance. Meaning of the symbols: $\tau_{1}$ - lifetime of the $\alpha$-Fe specimen, $\tau_{2,3}, I_{2,3}$ - lifetimes and intensities of the source contributions. Parameters of the resolution function: $w_{1}, w_{2}, \delta_{12}-$ FWHM's and separation of the first and the second Gaussian, the weight of the Gaussians was fixed at the ratio $I_{\mathrm{g} 1} /\left(I_{\mathrm{g} 1}+I_{\mathrm{g} 2}\right)=0.5, w$ - total FWHM of the resolution function (timing resolution). In case of the analogue setup, the resolution function contains an additional weak and broad Gaussian with FWHM $w_{3}$ and normalized intensity $I_{\mathrm{g} 3} .{ }^{a}$ The energy windows in the analogue setup were adjusted approximately at these energies using an oscilloscope; ${ }^{b}$ STOP-START mode, ${ }^{c}$ START-STOP mode The $\chi^{2}$ value reduced to number of degrees of freedom is shown in the last row. The errors (one standard deviation) are given in parentheses.

\begin{tabular}{|c|c|c|}
\hline Quantity & Digital setup & Analog setup \\
\hline \multicolumn{3}{|c|}{ Parameters of measurement } \\
\hline Count rate $\left[\mathrm{s}^{-1}\right]$ & 940 & 75 \\
\hline Accumulated coincidences & $200 \times 10^{6}$ & $10 \times 10^{6}$ \\
\hline Accepted coincidences $[\%]$ & 8.2 & 100 \\
\hline Effective count rate $\left[\mathrm{s}^{-1}\right]$ & 78 & 75 \\
\hline \multicolumn{3}{|c|}{ Energy windows } \\
\hline Stop energy window $[\mathrm{keV}]$ & $460-590$ & $\approx 450-600^{a}$ \\
\hline Start energy window $[\mathrm{keV}]$ & $1080-1550$ & $\approx 1100-1600^{a}$ \\
\hline \multicolumn{3}{|c|}{ Lifetimes and intensities } \\
\hline$\tau_{1}[\mathrm{ps}] \alpha$-Fe sample & $107.0(2)$ & $107.0(3)$ \\
\hline$\tau_{2}[\mathrm{ps}]-$ source & $365(4)$ & $370(5)$ \\
\hline$\tau_{3}[\mathrm{~ns}]$ - source & $1.29(5)$ & $1.35(3)$ \\
\hline$I_{2}[\%]$ & $8.45(7)$ & $8.7(2)$ \\
\hline$I_{3}[\%]$ & $1.23(5)$ & $1.24(4)$ \\
\hline \multicolumn{3}{|c|}{ Resolution function } \\
\hline$w_{1}[\mathrm{ps}]$ & $137.2(7)^{b} / 137(1)^{c}$ & $151.3(3)$ \\
\hline$w_{2}[\mathrm{ps}]$ & $155(1)^{b} / 153(1)^{c}$ & $187(1)$ \\
\hline$\delta_{12}[\mathrm{ps}]$ & $21(1)^{b} / 12(1)^{c}$ & $28(1)$ \\
\hline$w_{3}[\mathrm{ps}]$ & - & $600(30)$ \\
\hline$I_{\mathrm{g} 3}[\%]$ & - & $1.5(1)$ \\
\hline $\boldsymbol{w}[\mathrm{ps}]$ & $147^{b} / 145^{c}$ & 169 \\
\hline \multicolumn{3}{|c|}{ Quality of fit } \\
\hline$\chi^{2} / \nu$ & $1.01(2)$ & $1.07(2)$ \\
\hline
\end{tabular}




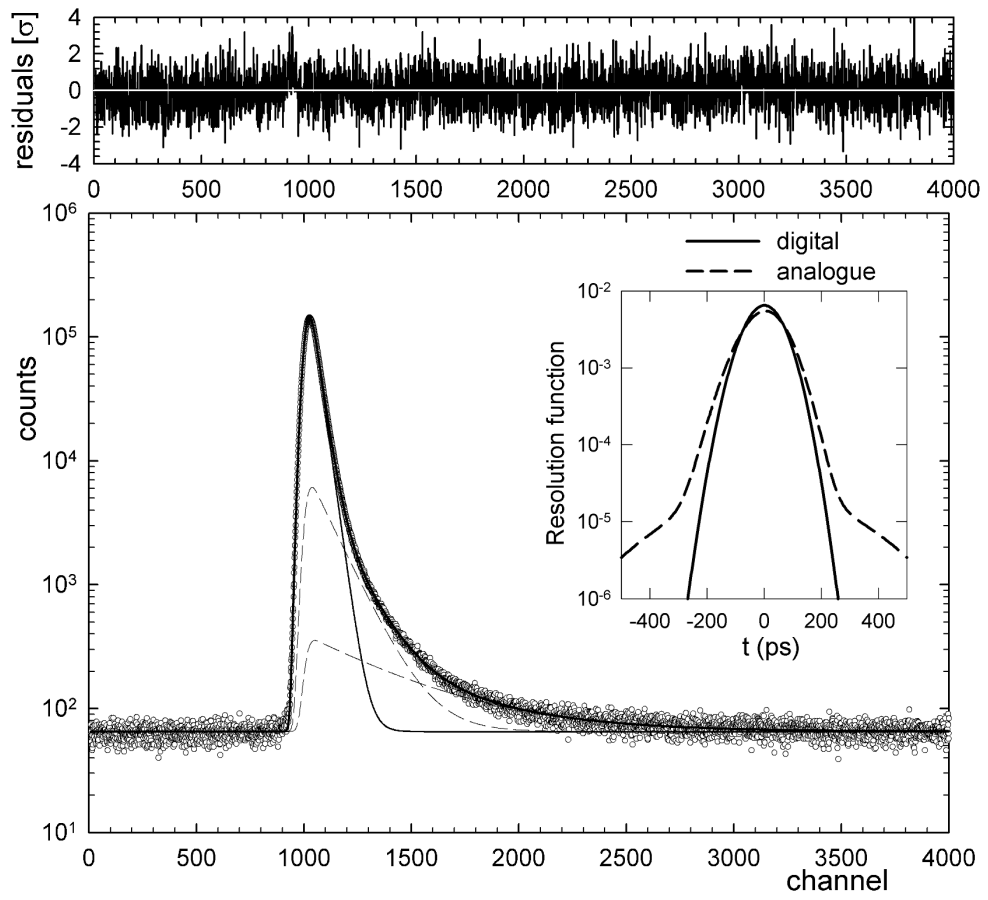

Fig. 2. Fit of the PL spectrum obtained in the digital setup (START-STOP mode). The resolution functions for the digital and the analogue setup are compared in the inset.

configuration. It clearly demonstrates that digital timing does improve timing resolution.

\section{Conclusions}

Performance of digital PL spectrometer equipped with two ultrafast digitizers was compared with the traditional analogue setup connected to the same detectors. The timing resolution of $146 \mathrm{ps}$ was achieved in the digital setup. It was demonstrated that the digital spectrometer can be operated in the START-STOP and STOP-START mode which doubles the count rate at no cost. Digital timing results in $\approx 20$ ps better timing resolution than analogue timing performed under similar conditions, keeping the count rate comparable.

\section{Acknowledgments}

This work is part of the research plan MS 0021620834 financed by the Ministry of Education of the Czech Republic. Financial support from the Czech Science Foundation (project No. 106/05/0073) is highly acknowledged. 


\section{References}

[1] J. Nissilä, K. Rytsölä, R. Aavikko, A. Laakso, K. Saarinen, P. Hautojärvi, Nucl. Instrum. Methods Phys. Res. A 538, 778 (2005).

[2] H. Saito, Y. Nagashima, T. Kurihara, T. Hyodo, Nucl. Intrum. Methods Phys. Res. A 487, 612 (2002).

[3] F. Bečvář, J. Čížek, L. Lešt'ák, I. Novotný, I. Procházka, F. Šebesta, Nucl. Instrum. Methods Phys. Res. A 443, 557 (2000).

[5] A. Seeger, F. Banhart, Phys. Status Solidi A 102, 171 (1987).

[4] F. Bečvář, Nucl. Instrum. Methods Phys. Res. B 261, 871 (2007). 